\title{
Synthetic Study toward a Protected 2-Deoxystreptamine
}

\author{
Seok-Chan Kim, ${ }^{*}$ Seung-Chul Lee, and Chan-Seong Cheong ${ }^{*}$ \\ Department of Bio \& Nano Chemistry, Kookmin University; Seoul 136-702, Korea \\ ${ }^{\dagger}$ Life Science Division. Korea Insitute of Science and Technology. P.O. Box 131, Cheongryang. Seoul 136-650. Korea \\ Received July 1. 2004
}

Key Words : 2-Deoxystreptamine, mo-lnositol, Stereoselective synthesis

2-Deoxystreplamine (1) is a key component of aminoglycoside antibiotics such as Streptomycin. Neomycins, Kanamycins, Gentamycins and Sisomycins which are still clinically useful. ${ }^{1.2}$ The structure and configuration were established to be a 1,3-diamino-4,5,6-cyclohexanctriol with all-irans configuration." Despite numerous rescarch interests in this area, only several chentical synthetic methods are known. ${ }^{4}$

I tere we described the first synthetic approach of the protected 2-deoxystreptamine from mo-inositol.

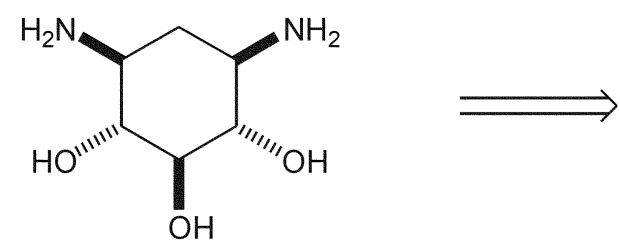

2-Deoxystreptamine (1)

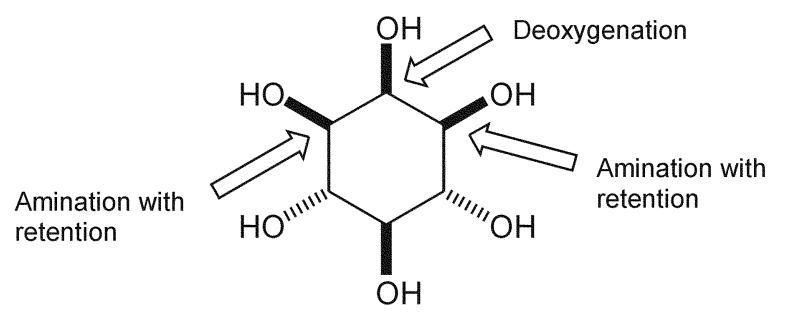

myo-Inositol

Reaction of myo-inositol with tricthyl ortholormate in the presence of acid catalyst gave inositol ortholomate 2 whose synthesis and structure was reported..$^{5}$ It provides simultaneous protection of three hydroxyl groups at C-1,3,5 and results in inversion of the axial/equatorial relationship of the remaining free hydroxyl groups (Scheme 1). Selective monobenzylation at $\mathrm{C}-4$ in $\mathbf{2}$ was cartied out with $\mathrm{Nal} I$ in DMF in high yield, together with a trace of the 4,6-dibenzyl ether 4. This high regioselectivity and degree of monobenzylation are presumably due 10 internal coordination in an intermediate anion." Radical induced cleavage method was employed to deoxygenate the OIl group at C-2 position. The less hindered equatorial C-2 hydroxyl group in 3 was selectively converted to xanthate ester compound $\mathbf{5}$, which was smoothly cleaved to 7 . The remaining C-6 position was subsequently protected with benzyl ether to alford 8. Iydrolysis of masking orthofomate group with aqueous IJCl provides 2-deoxy-4,6-O-dibenzyl-myo-inositol (9). Triols such as 9 are selectively prolected and masking of intermediate 10 with methyl ether aflords 12 . Fluorideassisted removal of silyl groups at C-1,3 allorded diol compound $\mathbf{1 3}$.

Introduction of amine function with the requisite conliguration at $\mathrm{C}-1,3$ positions was carricd out oxidation, oxime formation and reduction of oxime to amine sequences. Oxidation of 13 with PCC delivers monoketone compound which accompanies oxime formation with hydroxylamine in pyridine. Reduction of oxime 14 with $\mathrm{LiAlH}_{4}$ to anine compounds 15,16 procecded at a moderate pace and gave satisfactory yicld with an isomer ratio greater than $95: 5$ with $84 \%$ yicld. The successful outcome of this diastereoselectivity has been attributed to thermodynamically controlled reduction of oxime. If a chair conformation were favored for the reduction products 15, 16 from 14, the required isomer 16 with an equatorial amine substituent should be favored over 15 with that substituent axial (Scheme 2). Subsequent protection of resulting amine function in 16 with Boc group furnishes 17. Introduction of another amine function at C-3 position was accomplished by reiteration of above procedure with high stereoselectivity (92:8). Finally, protection of amine 19 with Boc group provides the target protected 2-deoxystreptamine 21 .

Stereochemical characterization of the target product 21 was accomplished by ${ }^{1} \mathrm{I}$ and ${ }^{13} \mathrm{C}$ NMR spectra. That the target 21 was the symmetrical isomer was readily apparent from the overlap of magnetic resonances corresponding to the equivalent hydrogens and carbons in its ' $I$ I NMR and ${ }^{15} \mathrm{C}$ NMR spectra." $\Lambda$ lthough several synthesis of the 2-deoxystreplamine were reported previously, the present synthesis of 21 is the lirst synthetic approach from myo-inositol as a starting material and, most importantly, generates the high stereoselectivity at C-1,3.

\section{Experimental Section}

( \pm ) 1,2-Dideoxy-1-amino-4,6-O-dibenzyl-5- $O$-methyl$m y o$-inositol (16). To a stirred solution of oxime $14(600$ mg. $1.62 \mathrm{mmol})$ in fireshly distilled THF $(10.0 \mathrm{~mL})$ was added $95 \% \mathrm{LiAlH}_{4}(260 \mathrm{mg}$. $6.48 \mathrm{mmol}$ ) at room temperature. The resulting mixture was refluxed for $2 \mathrm{~h}$. $\Lambda$ lier the 

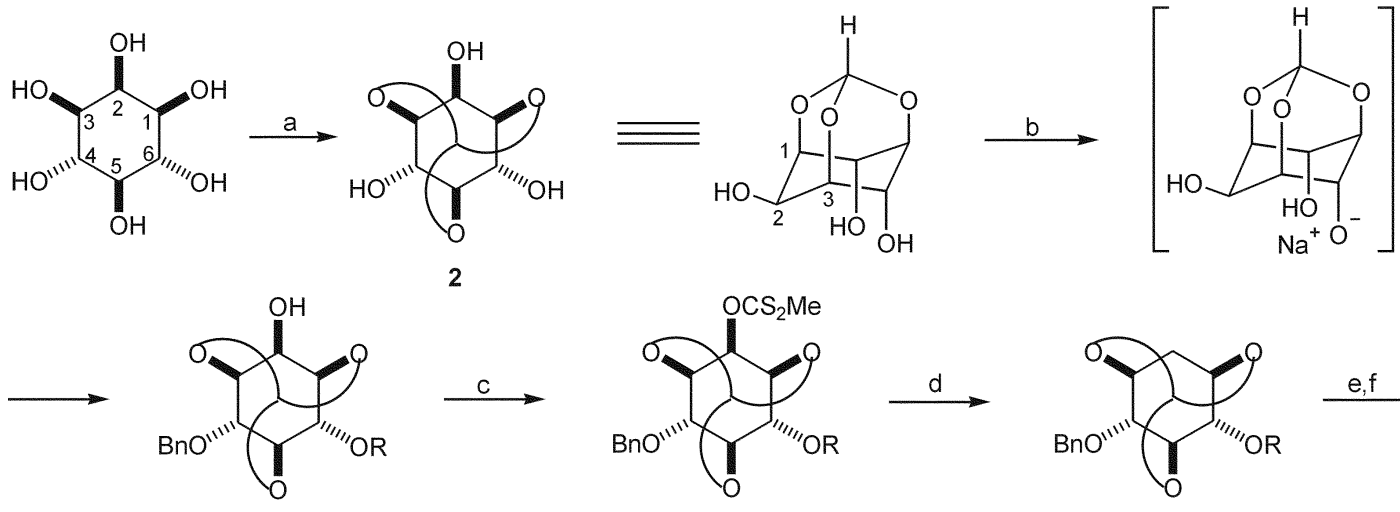

$$
\begin{aligned}
& \text { 3, } R=H \\
& 4, R=B n
\end{aligned}
$$

$5, \mathrm{R}=\mathrm{H}$

6, $\mathrm{R}=\mathrm{CS}_{2} \mathrm{Me}$

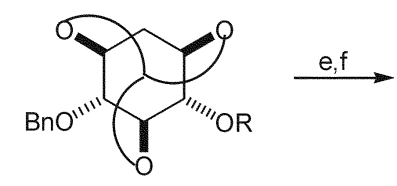

$7, \mathrm{R}=\mathrm{H}$

$8, R=B n$

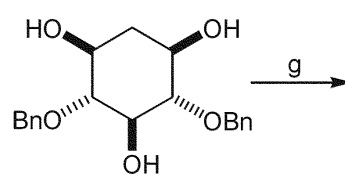

9<smiles>CCO[C@H]1C[C@H](OCC)[C@@H](OCc2ccccc2)[C@@H](O)[C@@H]1O</smiles>

$10, \mathrm{R}=\mathrm{H}$

$11, \mathrm{R}=\mathrm{TES}$<smiles>CCO[C@H]1C[C@@H](O[Si])[C@H](OCC)[C@H](OC)[C@@H]1OCc1ccccc1</smiles>

12

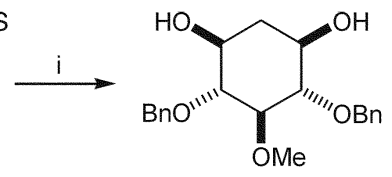

13

Scheme $1^{7}$. Reagents and conditions: (a) HC(OEt)s. $p$-TsOH (cat.). DMF, 80\%: (b) NaH (1.1 eq.). $\mathrm{BnBr}$ (1.1 eq.). DMF. $25^{\circ} \mathrm{C}, 85^{\circ}$ (3: 4

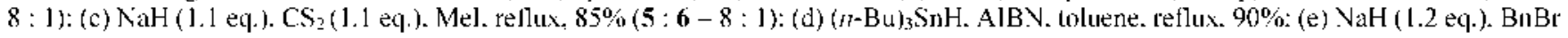

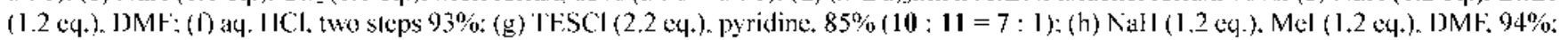
(i) $(n-\mathrm{Bu}) \mathrm{NT}$. ТHГ. $100 \%$.<smiles>CO[C@@H]1[C@@H](Cc2ccccc2)[C@H](O)C[C@@H](O)[C@@H]1OCc1ccccc1</smiles>

13<smiles>C[18CH][13CH3]</smiles>

14
14<smiles>CO[C@@H]1[C@@H](OCc2ccccc2)[C@@H](N)C[C@@H](N)[C@@H]1OCc1ccccc1</smiles>

$(8: 92)$<smiles>CO[C@@H]1[C@@H](OC(C)(C)C)[C@@H](N)C[C@@H](N)[C@@H]1OCc1ccccc1</smiles>

19<smiles>CO[C@@H]1[C@@H](OCc2ccccc2)[C@@H](OC(C)(C)C)C[C@@H](NC(=O)OCc2ccccc2)[C@H]1OCc1ccccc1</smiles>

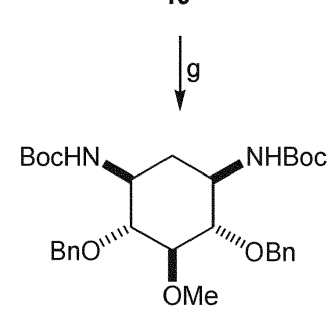

21<smiles>CO[C@H]1[C@@H](OCc2ccccc2)[C@H](N)C[C@H](O)[C@H]1Cc1ccccc1</smiles>

15<smiles>CO[C@H]1[C@@H](OCc2ccccc2)[C@H](O)C[C@H](N)[C@H]1Cc1ccccc1</smiles>

$(5: 95)$

16 d<smiles>CC(C)(C)OC(=O)N[C@H]1C[C@@H](O)[C@H](OCc2ccccc2)[C@H](OCc2ccccc2)[C@H]1OCc1ccccc1</smiles>

17

18

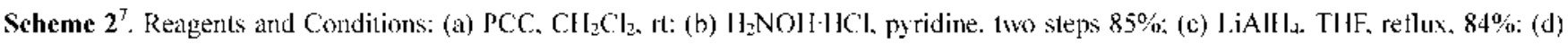

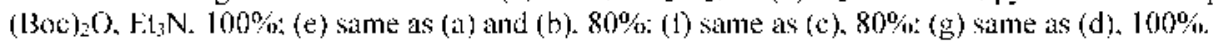

reaction mixture was cooled to room temperature, excess hydride was destroyed with $\mathrm{H}_{2} \mathrm{O}(1.0 \mathrm{~mL})$ and diluted with EtOAc $(25.0 \mathrm{~mL})$. The solution was filtered with a cake of florisil and concentrated under reduced pressure to afford crude product. This crude product was purified by column chromatography with $10 \% \mathrm{CH}_{2} \mathrm{Cl}_{2}$ in ethanol to give 16 (462 
mg): ${ }^{~} \mathrm{H}$ NMR $\left(\mathrm{CDCl}_{\mathrm{j} .} 300 \mathrm{MHz}\right) \delta 1.40(\mathrm{~m}, 2 \mathrm{H}), 3.19(\mathrm{~m}$. $1 \mathrm{H}), 3.41(\mathrm{~m}, \mathrm{lH}), 3.48(\mathrm{~m} . \mathrm{lH}) .3 .65(\mathrm{~m} . \mathrm{lH}) .3 .66(\mathrm{~s} .3 \mathrm{H})$. $4.02(\mathrm{~m}, \mathrm{lH}) .4 .68(\mathrm{~m} .3 \mathrm{H}), 5.00$ (d. $1 \mathrm{H}, J=11.4 \mathrm{~Hz}) .7 .35$ (m. 10H): ${ }^{13} \mathrm{C} \mathrm{NMR}\left(\mathrm{CDCl}_{3} .75 \mathrm{MHz}\right) \delta 34.01,46.70,61.95$. 68.01, 72.11. 75.50, 83.01, 83.04. 86.45, 128.63. 128.74 . 128.80. 128.91. 129.46, 129.51, 139.38, 139.50: Anal. Calcd for $\mathrm{C}_{21} \mathrm{H}_{37} \mathrm{NO}_{4}: \mathrm{C} .70 .56 ; \mathrm{H}, 7.61 ; \mathrm{N} .3 .92$. Found: $\mathrm{C}, 70.47$; H. 7.58: $\mathrm{N}, 3.9 \mathrm{I}$.

(士) 1,2-Dideoxy-1- $N$-Boc-4,6-O-dibenzyl-5- $O$-methylmyo-inositol (17). Di-tert-butyl dicarbonate (135 $\mathrm{mg}, 0.62$ inmol) in THF ( $1.5 \mathrm{~mL}$ ) was added dropwise over $10 \mathrm{~min}$. to a stirred solution of freshly distilled THF $(2.6 \mathrm{~mL})$, anine compound 16 (148 mg. 0.414 mmol) and $\mathrm{Et}_{3} \mathrm{~N}(0.36 \mathrm{~mL})$ at roon temperature under $\mathrm{N}_{\mathrm{s}}$. This resulting mixture was stirred at room temperature for $3 \mathrm{~h}$ and quenched with $\mathrm{H}_{3} \mathrm{O}$ $(0.5 \mathrm{~mL})$. The solution was extracted with EtOAc $(2 \times 10.0$ $\mathrm{mL}$ ) and organic layer was rinsed with brine. dried over anhydrous $\mathrm{MgSO}_{4}$. filtered and concentrated under reduced pressure to give crude product. This crude product was purified by column chromatograph with $20 \% \mathrm{EtOAc}$ in hexane to give compound 17 (187 mg): ${ }^{~} \mathrm{H} \mathrm{NMR}\left(\mathrm{CDCl}_{3}\right.$. $300 \mathrm{MHz}) \delta 1.39$ (s. 9H), 1.44 (m. $2 \mathrm{H}$ ). 3.23 (dd. $1 \mathrm{H} . J=8.4$ \& $10.5 \mathrm{~Hz}) .3 .32(\mathrm{~m}, \mathrm{lH}), 3.52(\mathrm{~m}, \mathrm{lH}), 3.62(\mathrm{~s} .3 \mathrm{H}) .3 .73$ (m. IH). $4.15(\mathrm{~m}, \mathrm{lH}) .4 .55$ (d. IH. $J=11.4 \mathrm{~Hz}) .4 .64$ (d. $1 \mathrm{H}, J=11.4 \mathrm{~Hz}), 4.68(\mathrm{~d} .1 \mathrm{H}, J=11 .+\mathrm{Hz}) .4 .93(\mathrm{~d}, 1 \mathrm{H}, J=$ $11.4 \mathrm{~Hz}) .7 .35$ (m. $10 \mathrm{H}) ;{ }^{13} \mathrm{C}$ NMR $\left(\mathrm{CDCl}_{3}, 75 \mathrm{MHz}\right) \delta$ $29.35,32.09$. 46.00, 60.50, 61.78, 68.00, 71.80, 75.02. $80.10,83.59,84.50,128.74 .128 .77,128.80,128.91$. 129.46. 129.51. 139.21, 139.39, 156.38; Anal. Calcd for $\mathrm{C}_{36} \mathrm{H}_{3 \leq} \mathrm{NO}_{6}$ : C. $68.25: \mathrm{H}, 7.7 \mathrm{I}:$ N. 3.06 . Found: C. $69.07 ; \mathrm{H}, 7.78: \mathrm{N}$. 3.09 .

(土) 1- $\mathrm{N}$-Boc-4,6-O-dibenzyl-5- $O$-methyl-2-deoxystreptamine (19). ${ }^{~} \mathrm{H}$ NMR $\left(\mathrm{CDCl}_{3}, 300 \mathrm{MHz}\right) \delta \mathrm{l} .40$ (s. $9 \mathrm{H}$ ). $1.70(\mathrm{~m} .2 \mathrm{H}) .3 .25(\mathrm{~m}, \mathrm{lH}), 3.26(\mathrm{~s}, 3 \mathrm{H}), 3.50(\mathrm{~m} .3 \mathrm{H}) .4 .10$ (m. $1 \mathrm{H}), 4.63(\mathrm{~m} .4 \mathrm{H}), 4.55$ (d. $1 \mathrm{H}, J=11.4 \mathrm{~Hz}) .7 .31(\mathrm{~m}$. 10H): ${ }^{13} \mathrm{C}$ NMR $\left(\mathrm{CDCl}_{3} .75 \mathrm{MHz}\right) \delta 29.02 .29 .96 .41 .74$.
$48.90,68.39 .72 .32 .72 .63 .73 .02,77.25,77.69 .127 .77$, $128.77,128.00 .128 .11,128.52,128.64 .128 .90 .138 .49$. 138.87, 155.68: Anal. Calcd for $\mathrm{C}_{26} \mathrm{H}_{36} \mathrm{~N}_{2} \mathrm{O}_{5}: \mathrm{C}, 68.40 ; \mathrm{H}$, $7.95 ;$ N. 6.14. Found: C. $68.77 ;$ H. 7.89 : N, 6.19.

(土) 1,3-Di- $N, N^{\prime}$-Boc-4,6-O-dibenzyl-5- $O$-methyl-2-deoxystreptamine (21). ${ }^{1} \mathrm{H}$ NMR $\left(\mathrm{CDCl}_{3} .300 \mathrm{MHz}\right) \delta 1.42$ (s. $18 \mathrm{H}), 1.60(\mathrm{~m}, 2 \mathrm{H}), 3.25(\mathrm{~s}, 3 \mathrm{H}) .3 .57(\mathrm{~m}, \mathrm{lH}) .3 .64(\mathrm{~m}$, $2 \mathrm{H}$ ). 3.89 (m. $2 \mathrm{H}$ ). 4.50 (d. $2 \mathrm{H} . J=11.4 \mathrm{~Hz}$ ). 4.62 (d. $2 \mathrm{H} . J=$ $11.7 \mathrm{~Hz}), 7.30(\mathrm{~m}, 10 \mathrm{H}):{ }^{13} \mathrm{C}$ NMR $\left(\mathrm{CDCl}_{3}, 75 \mathrm{MHz}\right) \delta$ $28.30,29.01,47.74 .58 .45 .72 .11,76.90$. 77.56. 79.61, 128.10, 128.25. 128.70. 138.51, 156.38. Anal Calcd for $\mathrm{C}_{31} \mathrm{H}_{44} \mathrm{~N}_{2} \mathrm{O}_{7}: \mathrm{C}$. 66.88; H. 7.97: N, 5.03. Found: $\mathrm{C}, 66.67: \mathrm{H}$, 7.87: N. 5.09 .

Acknowledgment. We would like to thank Korea Institute of Science and Technology for financial support of this work.

\section{References and Note}

1. (a) Kuehl. F. A.: Bishop. M. N.: Folkers. K. $J$. Am. Chom. Soc. 1951. 73. 881. (b) Umezawa. H.: Ueda. M: Maeda. K. J. Antibiotic 1957. 10.1, 181.

2. (a) Hoffhime. P; Gale. F. J. Am. Chen. Soc, 1949. 71, 2590. (b) Haskell, T. H.: French. J. C.: Bartz. Q. R. J. Am. Chem. Soc. 1959. 81.3480 .

3. Lemieux. R. U.: Cushley. R. J. Can. J. Chem. 1963. H1. 858.

4. (a) Nakajima. M.: Hasegawa. A.: Kurihara. N. Tetrohedron Lett. 1964. 17. 967. (b) Suami. T.; Lichtenthaler, F. W.: Ogawa. S: Nakashima. Y: Sano. H Bull Chem. Soc. Jpm 1967. 40,1014. (c) Silva, E. T.: Hyaric, M. L.: Machdado, M. V: Mauro. V. A. Tetrahedron Lett 1998. 39.6659 .

5. Kishi. Y.: Lee. H. W. J. Ong Chem 1986. 50.4402.

6. Billington. D. C.: Baker. R.: Kulagowski. I. J.: Mawer. I. M.: Vaca, J. P.: deSolms. S. J.; Huff. J. R. J. Chent Soc. Perkin Trans. I 1989,1423 .

7. All compounds are drawn as their absolute configuration but are racemic mixtures.

8. See the $\mathbf{n m r}$ data of compound 21 in experimental section. 